\title{
A model independent observer based output feedback tracking controller for robotic manipulators with dynamical uncertainties Erkan Zergeroglu $\dagger^{*}$, Enver Tatlicioglu $\ddagger$ and Egemen Kaleli†
}

\author{
$\dagger$ Department of Computer Engineering, Gebze Institute of Technology, 41400, Gebze, Kocaeli, \\ Turkey.E-mail: ekaleli@bilmuh.gyte.edu.tr \\ $\ddagger$ Department of Electrical \& Electronics Engineering, Izmir Institute of Technology, Gulbahce \\ Koyu, Urla, Izmir, 35430 Turkey.E-mail: envertatlicioglu@iyte.edu.tr
}

(Accepted September 9, 2015. First published online: October 5, 2015)

\begin{abstract}
SUMMARY
In this work, we propose the development and the corresponding stability analysis of a novel, observer-based output feedback (OFB), tracking controller for rigid-link robot manipulators. Specifically, a model-independent variable-structure-like observer in conjunction with a desired dynamic compensation technique have been utilized to remove the link velocity dependency of the controller formulation. Asymptotic stability of the observer-controller couple is then guaranteed via Lyapunov-based arguments. An adaptive controller extension is also presented to illustrate the expansiveness of the proposed scheme. Experimental studies performed on a two-link planar robot with dynamical uncertainties are included in order to demonstrate the performance and feasibility of the proposed method.
\end{abstract}

KEYWORDS: Output feedback Control; Observer Based Design; Adaptive Systems.

\section{Introduction}

Controlling robotic manipulators using only position measurements has been a relatively attractive research field. Aside from the challenging nature of the problem, the main reason for this interest is due to the reduction of velocity sensors. Most robots available for industrial use, do not have velocity sensors. And sensors like tachometers, mostly, are contaminated with noise. Therefore, removing the need to use them in the controller design, is attractive from an implementation point of view. Extracting the velocity information via numerical differentiation plus low pass filtering is a common approach used in full state feedback controller formulations, however this approach introduces extra dynamical terms in the closed loop system, which have to be accounted for in the stability analysis. Therefore, construction of velocity surrogate signals that can be used for the closed-loop operation is a well-motivated research field. To our best knowledge; existing solutions to the OFB tracking

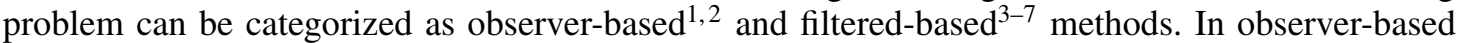
methods, mostly a model-dependent observer ${ }^{2,8}$ is used to estimate the velocity signal. On the other hand, in filtered-based approaches, surrogate signals obtained via some means of filtering approaches are used to overcome the need of velocity measurements. This work concentrates on the design of a new model-free observer-based OFB controller formulation.

Some of the past research that applied a similar approach are as follows: In ref. [9], a variablestructure OFB controller was designed to compensate for the lack of link velocity measurement. Similarly in ref. [10], a discontinuous controller with a high gain observer was proposed for the stabilization of a class of non-linear systems. Recently, a Luenberger like observer with an extra switching term was proposed in ref. [11] for the OFB control of robot manipulators.

* Corresponding author. E-mail: ezerger@ bilmuh.gyte.edu.tr 
In this paper, inspired by the observer structure given in ref. [12], we propose a new model free observer in conjunction with a desired robot model-based controller formulation for the output feed tracking control of robot manipulators. The observer/controller structure proposed achieves semi-global asymptotic tracking despite the lack of velocity measurements.

The novelty of the proposed observer-controller lies in the simplicity of the overall design and also the fact that using some mathematical manipulations, the proposed OFB controller formulation can be written in a full state feedback controller form (details are given in Section 4). This enables the overall design to be modular. That is; most of the full state controller proposed for robot manipulators can be extended to OFB form using the proposed observer-controller formulation with a considerable small effort. In order to illustrate the modularity of the proposed controller, an adaptive version where the dynamical parameters of the robot manipulator are assumed to be uncertain is presented here. The adaptive extension of the proposed method achieves semi-global asymptotic tracking. It is our belief that robust and learning type OFB versions of the proposed method are also possible.

The rest of the work ${ }^{1}$ is organized in the following manner: In Section 2, the dynamical model of the robot manipulator with its properties used in the analysis and design of the proposed observercontroller couple are presented while Section 3 contains the error system development and problem formulation. In Section 4, the design and stability analysis of the controller and observer are proposed. The adaptive controller extension is presented in Section 5. In Section 6, we demonstrate the effectiveness of the proposed method through simulation results obtained from a two link, direct drive planar robot manipulator. Concluding remarks are presented in Section 7.

\section{System Model and Dynamical Properties}

The mathematical model for an $n$ DOF, revolute joint, direct drive robot manipulator is assumed to have the following form: ${ }^{13}$

$$
M(q) \ddot{q}+V_{m}(q, \dot{q}) \dot{q}+G(q)+F_{d} \dot{q}=\tau,
$$

where $q(t), \dot{q}(t), \ddot{q}(t) \in \mathbb{R}^{n}$ denote the link position, velocity, and acceleration, respectively, $M(q) \in$ $\mathbb{R}^{n \times n}$ represents the positive-definite, symmetric inertia matrix, $V_{m}(q, \dot{q}) \in \mathbb{R}^{n \times n}$ represents the centripetal-Coriolis matrix, $G(q) \in \mathbb{R}^{n}$ is the gravitational vector, $F_{d} \in \mathbb{R}^{n \times n}$ denotes the constant, diagonal, positive-definite viscous friction matrix, and $\tau(t) \in \mathbb{R}^{n}$ represents the torque input control vector. In the subsequent development, we will assume that the left-hand side of Eq. (1) is first-order differentiable.

The dynamic model given by Eq. (1) exhibits the following properties that will be utilized in the subsequent control development and the associated stability analysis.

Property 1. The inertia matrix can be bounded from above and below by the following inequalities: ${ }^{13}$

$$
m_{1} I_{n} \leq M(q) \leq m_{2} I_{n},
$$

where $m_{1}$ and $m_{2}$ are positive constants, and $I_{n}$ is the standard $n \times n$ identity matrix. Likewise, the inverse of the inertia matrix can be bounded as follows: ${ }^{13}$

$$
\frac{1}{m_{2}} I_{n} \leq M^{-1}(q) \leq \frac{1}{m_{1}} I_{n} .
$$

Property 2. The inertia and the centripetal-Coriolis matrices satisfy the following relationship: ${ }^{14}$

$$
\xi^{T}\left(\frac{1}{2} \dot{M}(q)-V_{m}(q, \dot{q})\right) \xi=0 \quad \forall \xi \in \mathbb{R}^{n},
$$

where $\dot{M}(q)$ represents the time derivative of the inertia matrix.

\footnotetext{
${ }^{1}$ Preliminary version of this work appeared in refs. [15] and [16].
} 
Property 3. The centripetal-Coriolis matrix satisfies the following relationship: ${ }^{8}$

$$
V_{m}(q, v) \xi=V_{m}(q, \xi) \nu \quad \forall \xi, v \in \mathbb{R}^{n} .
$$

Property 4. The norm of the centripetal-Coriolis and friction matrices can be upper bounded as follows: ${ }^{13}$

$$
\left\|V_{m}(q, \xi)\right\| \leq \zeta_{c 1}\|\xi\|, \quad\left\|F_{d}\right\| \leq \zeta_{f} \quad \forall \xi \in \mathbb{R}^{n},
$$

where $\zeta_{c 1}$ and $\zeta_{f}$ are positive constants.

Property 5. The robot dynamics given in Eq. (1) can be linearly parameterized as follows: ${ }^{13}$

$$
Y(q, \dot{q}, \ddot{q}) \theta=M(q) \ddot{q}+V_{m}(q, \dot{q}) \dot{q}+G(q)+F_{d} \dot{q},
$$

where $\theta \in \mathbb{R}^{p}$ contains the constant system parameters, and $Y(q, \dot{q}, \ddot{q}) \in \mathbb{R}^{n \times p}$ denotes the regression matrix that is a function of only $q(t), \dot{q}(t)$, and $\ddot{q}(t)$. The formulation of Eq. (7) can also be written in terms of the desired trajectory in the following manner:

$$
Y_{d}\left(q_{d}, \dot{q}_{d}, \ddot{q}_{d}\right) \theta=M\left(q_{d}\right) \ddot{q}_{d}+V_{m}\left(q_{d}, \dot{q}_{d}\right) \dot{q}_{d}+G\left(q_{d}\right)+F_{d} \dot{q}_{d},
$$

where the desired regression matrix $Y_{d}\left(q_{d}, \dot{q}_{d}, \ddot{q}_{d}\right) \in \mathbb{R}^{n \times p}$ is a function of the desired link position, velocity, and acceleration vectors, denoted by $q_{d}(t), \dot{q}_{d}(t), \ddot{q}_{d}(t) \in \mathbb{R}^{n}$, respectively.

Property 6. The inertia, centripetal-Coriolis, and gravity terms in Eq. (1) can be upper bounded as follows: ${ }^{17}$

$$
\begin{gathered}
\|M(\xi)-M(v)\|_{i \infty} \leq \zeta_{m 1}\|(\xi-v)\| \\
\left\|M^{-1}(\xi)-M^{-1}(v)\right\|_{i \infty} \leq \zeta_{m 2}\|(\xi-v)\| \\
\left\|V_{m}(\xi, \eta)-V_{m}(v, \eta)\right\|_{i \infty} \leq \zeta_{c 2}\|\eta\|\|(\xi-v)\| \\
\|G(\xi)-G(v)\| \leq \zeta_{g}\|(\xi-v)\|
\end{gathered}
$$

$\forall \xi, \nu, \eta \in \mathbb{R}^{n}$, where $\zeta_{m 1}, \zeta_{m 2}, \zeta_{c 2}$, and $\zeta_{g} \in \mathbb{R}$ are positive bounding constants, and $\|\cdot\|_{i \infty}$ denotes the induced infinity norm of a matrix.

\section{Problem Formulation}

The control objective is to design a link position tracking controller for the robot manipulator model given by Eq. (1) under the constraints that only the link position variable $q(t)$ is available for measurement. We will also assume that the parameter vector $\theta$ defined in Eq. (6) is a constant vector, that is the parameters of the overall system are not changing in time.

We will quantify the control objective by defining the link position tracking error $e(t) \in \mathbb{R}^{n}$ as follows:

$$
e \triangleq q_{d}-q
$$

where we assume that $q_{d}(t)$, defined in Property 5 , and its first three time derivatives are bounded functions of time. To account for the unmeasurable link velocity constraint, we define $\dot{\hat{q}} \in \mathbb{R}^{n}$ as the observed velocity signal. The corresponding velocity and position observation error signals $\dot{\tilde{q}}, \tilde{q} \in \mathbb{R}^{n}$ are defined as

$$
\begin{aligned}
& \dot{\tilde{q}}=\dot{q}-\dot{\hat{q}}, \\
& \tilde{q}=q-\hat{q} .
\end{aligned}
$$

To ease the presentation of the analysis, we will use two auxiliary variables, filtered tracking error $r(t) \in \mathbb{R}^{n}$, and filtered observation error like term $s(t) \in \mathbb{R}^{n}$ as

$$
r \triangleq \dot{e}+\alpha e, \text { and } s \triangleq \dot{\tilde{q}}+\alpha \tilde{q}
$$


where the controller gain matrix $\alpha \in \mathbb{R}_{+}$is a positive control gain. Notice that regulating $r(t)$ and $s(t)$ ensures the regulation of $e(t)$ and $\tilde{q}(t)$ respectively.

\section{Observer-Controller Design}

Based on the subsequent error system development and the stability analysis, we propose the following velocity observer:

$$
\begin{aligned}
& \dot{\hat{q}}=p+K_{0} \tilde{q}-K_{c} e \\
& \dot{p}=K_{1} \operatorname{Sgn}(\tilde{q})+K_{2} \tilde{q}-\alpha K_{c} e,
\end{aligned}
$$

where $p(t)$ is an auxiliary variable, $\operatorname{Sgn}(\cdot)$ is defined as

$$
\operatorname{Sgn}(\zeta)=\left[\begin{array}{llll}
\operatorname{sgn}\left(\zeta_{1}\right) & \operatorname{sgn}\left(\zeta_{2}\right) & \ldots \operatorname{sgn}\left(\zeta_{n}\right)
\end{array}\right]^{T} \quad \curlyvee \zeta \in \mathbb{R}^{n}
$$

with $\operatorname{sgn}(\cdot)$ being the sign function, $K_{0}, K_{c}, K_{1}, K_{2} \in \mathbb{R}^{n \times n}$ are diagonal, positive define gain matrices and $\alpha \in \mathbb{R}_{+}$was defined in Eq. (12).It is straightforward to show that the time derivative of Eq. (13) yields

$$
\ddot{\hat{q}}=K_{1} \operatorname{Sgn}(\tilde{q})+K_{2} \tilde{q}+K_{0} \dot{\tilde{q}}-K_{c} r,
$$

where the definition of $r(t)$ given in Eq. (12) has been utilized. Similarly, assuming that exact knowledge of all the system parameters are available, the control torque input signal $\tau(t)$ is designed to have the following form:

$$
\tau=Y_{d} \theta+K_{p} e+K_{c} \alpha\left(q_{d}-\hat{q}\right)+K_{c}\left(\dot{q}_{d}-\dot{\hat{q}}\right),
$$

where the first term, $Y_{d} \theta$, defined in Eq. (8) is the desired robot dynamics, $K_{p} \in \mathbb{R}^{n \times n}$ is diagonal positive define control gain matrix and $K_{c}, \alpha$ were previously defined. Note that using the fact that

$$
q_{d}-\hat{q}=e+\tilde{q}
$$

the control torque input given in Eq. (16) can be re-written in the following advantageous form:

$$
\tau=Y_{d} \theta+K_{p} e+K_{c} r+K_{c} s .
$$

\subsection{Observer analysis}

Utilizing Eq. (1) for the link acceleration signal and Eq. (15) for the time derivative of the observed velocity signal, the observation error dynamics can be obtained as

$$
\begin{aligned}
\ddot{\tilde{q}} & =\ddot{q}-\ddot{\hat{q}} \\
& =N_{0}-K_{1} \operatorname{Sgn}(\tilde{q})-K_{2} \tilde{q}-K_{0} \dot{\tilde{q}}+K_{c} r,
\end{aligned}
$$

where the auxiliary term $N_{0}$ is defined as

$$
N_{0}=M^{-1}(q)\left\{\tau-V_{m}(q, \dot{q}) \dot{q}-G(q)-F_{d} \dot{q}\right\} .
$$

Inserting Eqs.(18) and (8) in Eq. (20), we can explicitly obtain the following expression:

$$
N_{0}=N_{d}+N_{b},
$$

where the auxiliary variables $N_{d}$ and $N_{b}$ are specifically defined as

$$
N_{d}(t) \triangleq \ddot{q}_{d}
$$


and

$$
\begin{aligned}
N_{b}(t) \triangleq & \left(M^{-1}(q)-M^{-1}\left(q_{d}\right)\right) M\left(q_{d}\right) \ddot{q}_{d} \\
& +M^{-1}(q)\left\{V_{m}\left(q_{d}, \dot{q}_{d}\right) \dot{q}_{d}-V_{m}(q, \dot{q}) \dot{q}\right. \\
& +G\left(q_{d}\right)-G(q)+F_{d}\left(\dot{q}_{d}-\dot{q}\right) \\
& \left.+K_{p} e+K_{c}(r+s)\right\} .
\end{aligned}
$$

Exploiting the boundedness properties of the desired trajectory, we can show that both $N_{d}(t)$ and its time derivative, $\dot{N}_{d}(t)$, are bounded. Furthermore, as illustrated in Appendix I, using the properties (5), (6), (9), and mean value theorem $N_{b}$ of Eq. (23) can be upper bounded as follows:

$$
\left\|N_{b}(t)\right\| \leq \rho_{o 1}\|e\|+\rho_{o 2}\|r\|+\rho_{o 3}\|r\|^{2}+\rho_{o 4}\|s\|,
$$

where $\rho_{o i}, i=1, \ldots, 4$ are some positive known bounding functions that depend on the mechanical parameters and the desired trajectory and $\|\cdot\|$ denotes the Euclidean norm.

Taking the time derivative $s(t)$ of Eq. (12) and inserting for Eq. (19), the dynamics for the filtered observation error $s(t)$, can be obtained as follows:

$$
\dot{s}=N_{d}+N_{b}-K_{1} \operatorname{Sgn}(\tilde{q})-K_{2} \tilde{q}-\left(K_{0}-\alpha\right) \dot{\tilde{q}}+K_{c} r
$$

and when the observer gains are selected to satisfy

$$
\alpha\left(K_{0}-\alpha\right)=K_{2}
$$

Eq. (25) can be rearranged to have the following form:

$$
\dot{s}=N_{d}+N_{b}-K_{1} \operatorname{Sgn}(\tilde{q})-\frac{K_{2}}{\alpha} s+K_{c} r
$$

which enables us to state the following preliminary Lyapunov-like analysis for the observer. Specifically, we define the following non-negative scalar function $V_{o}(t)$, as follows:

$$
V_{0}=\frac{1}{2} s^{T} s+P_{0}
$$

where the scaler auxiliary function $P_{0}(t)$ is defined as

$$
P_{0}=\zeta_{0}-\int_{t_{0}}^{t} w_{0}(\sigma) d \sigma
$$

with the scalar function $w_{0}$ and the non-negative constant $\zeta_{0}$ defined as

$$
\begin{gathered}
w_{0} \triangleq s^{T}\left[N_{b}-K_{1} \operatorname{Sgn}(\tilde{q})\right] \\
\zeta_{0} \triangleq \sum_{i=0}^{n} K_{1 i}\left|\tilde{q}_{i}(0)\right|-\tilde{q}^{T}(0) N_{d}(0),
\end{gathered}
$$

where the subscript $i=1,2, \ldots, n$ denotes the $i^{t h}$ element of the vector or diagonal matrix. Following a similar analysis to that of refs. $[12,18]$, also presented in Appendix II, it can be proven that when $K_{1}$ satisfies the following sufficient condition:

$$
K_{1 i}>\left\|N_{d i}(t)\right\|_{\infty}+\frac{1}{\alpha}\left\|\dot{N}_{d i}(t)\right\|_{\infty}
$$

where the subscript $i=1,2, \ldots, n$ denotes the $i^{\text {th }}$ element of the diagonal matrix and $\|\cdot\|_{\infty}$ denotes the $\mathcal{L}_{\infty}$ norm, then $P_{0}(t)$ of Eq. (29) is always non-zero, that is $P_{0}(t) \geq 0$ and $V_{0}$ is a positive definite 
Lyapunov function with respect to $s$ and $\sqrt{P_{0}}$. Taking the time derivative of Eq. (28) and substituting for Eq. (27), time derivative of Eqs.(29) and (30), we obtain

$$
\dot{V}_{0}=s^{T}\left[-\frac{K_{2}}{\alpha} s+K_{c} r+N_{b}\right] .
$$

The first term in the brackets of Eq. (32) will be used for both damping the unwanted effects of the term $N_{b}$ in the composite stability analysis and to ensure the convergence of the observation error. The second term is designed to cancel out the interconnection term between the observer/controller subsystem. At this point, we are ready to proceed to the error system development.

\subsection{Error system development}

To obtain the dynamics of $r(t)$, we take its time derivative and premultiply the resulting equation by $M(q)$, utilizing Eqs.(1) and (10) and perform some algebraic manipulation, to produce

$$
M(q) \dot{r}=-V_{m}(q, \dot{q}) r+Y_{s} \theta-\tau,
$$

where the auxiliary term $Y_{s} \theta \in \mathbb{R}^{n}$ is defined as

$$
Y_{s} \theta=M(q)\left(\ddot{q}_{d}+\alpha \dot{e}\right)+V_{m}(q, \dot{q})\left(\dot{q}_{d}+\alpha e\right)+G(q)+F_{d} \dot{q} .
$$

After substituting the control law Eq. (18) into Eq. (33), we obtain the following closed-loop dynamics for $r(t)$

$$
M(q) \dot{r}=-V_{m}(q, \dot{q}) r+\chi-K_{c} r-K_{c} s-K_{p} e,
$$

where the disturbance term $\chi(r, e, t) \in \mathbb{R}^{n}$ is defined as follows:

$$
\chi=Y_{s} \theta-Y_{d} \theta
$$

with $Y_{d} \theta$ term defined in Eq. (8).

As illustrated in ref. [13], and also shown in Appendix I, we can exploit the boundedness properties of the desired trajectory and the Eqs.(5), (6), and (9) properties of the robot dynamics to show that the norm of the variable $\chi(\cdot)$ defined in Eq. (36) can be upper bounded as

$$
\|\chi\| \leq \rho_{1}(e)\|e\|+\rho_{2}(e)\|r\|,
$$

where $\rho_{1}(e)$ and $\rho_{2}(e)$ are positive known bounding functions. The above bound will be exploited to obtain the stability result presented in the next section.

\subsection{Stability analysis}

The combination of error systems for Eqs.(27) and (35) yields the following stability result for the observation error and the position tracking error.

Theorem 1. The velocity observer of Eq. (13) and the control law of Eq. (16) ensure that the closed-loop observer/controller is semi-globally asymptotically stable in the sense that

$$
\lim _{t \rightarrow \infty} e(t), \dot{\tilde{q}}(t)=0,
$$

provided that the controller and observer gains are selected to satisfy Eqs.(26), (31) with controller gain $K_{c}$ observer gain $K_{2}$ are defined as follows:

$$
\begin{aligned}
& K_{c}=\left(1+\rho_{2}+k_{n} \rho_{1}^{2}\right) I_{n} \\
& K_{2}=\alpha\left(1+\rho_{o 4}+k_{n}\left(\rho_{o 1}^{2}+\rho_{o 2}^{2}+\rho_{o 3}^{2}\right)\right) I_{n},
\end{aligned}
$$


where $\rho_{1}(e), \rho_{2}(e)$ were defined in Eq. (37), $\rho_{o i}, i=1,2,3,4$ were defined in Eq. (24) and $k_{n}$ being a non-linear damping gain selected to satisfy the following condition:

$$
k_{n}>\left(1+\frac{\lambda_{2}}{\lambda_{1}}\|z(0)\|^{2}\right) / 2
$$

and $z(t)$ defined as follows:

$$
z(t) \triangleq\left[\begin{array}{llll}
s^{T} & \sqrt{P_{0}} & r^{T} & e^{T}
\end{array}\right]^{T}
$$

and the positive bounding constants $\lambda_{1}$ and $\lambda_{2}$ are defined as

$$
\begin{gathered}
\lambda_{1}=\frac{1}{2} \min \left\{1, m_{1}, \lambda_{\min }\left\{K_{p}\right\}\right\} \\
\lambda_{2}=\frac{1}{2} \max \left\{1, m_{2}, \lambda_{\max }\left\{K_{p}\right\}\right\} .
\end{gathered}
$$

Proof. We start our proof by introducing the following non-negative function in the form

$$
V=V_{0}+\frac{1}{2} r^{T} M(q) r+\frac{1}{2} e^{T} K_{p} e .
$$

From Eq. (43), $V(t)$ can be upper and lower bounded as

$$
\lambda_{1}\|x\|^{2} \leq \lambda_{1}\|z\|^{2} \leq V \leq \lambda_{2}\|z\|^{2},
$$

where $x(t) \in \mathbb{R}^{3 n}$ is defined as

$$
x(t) \triangleq\left[\begin{array}{lll}
s^{T} & r^{T} & e^{T}
\end{array}\right]^{T},
$$

$z(t)$ were defined in Eq. (41) and the positive constants $\lambda_{1}, \lambda_{2}$ were defined in Eq. (42). Differentiating Eq. (43) with respect to time, substituting for the error systems introduce in Eqs.(32), (35) and cancelling common terms produces

$$
\dot{V}=s^{T}\left[-\frac{K_{2}}{\alpha} s+N_{b}\right]+r^{T}\left[\chi-K_{c} r\right]-\alpha K_{p}\|e\|^{2},
$$

where property (4) has been utilized. After applying Eqs.(24) and (36) to Eq. (46), we can form an upper bound on $\dot{V}(t)$ as follows:

$$
\begin{aligned}
\dot{V} \leq & -\|e\|^{2}-\|r\|^{2}-\|s\|^{2} \\
& +\left[\rho_{o 1}\|e\|\|s\|-k_{n} \rho_{o 1}^{2}\|s\|^{2}\right] \\
& +\left[\rho_{o 2}\|r\|\|s\|-k_{n} \rho_{o 2}^{2}\|s\|^{2}\right] \\
& +\left[\rho_{o 3}\|r\|^{2}\|s\|-k_{n}^{2} \rho_{o 3}^{2}\|s\|^{2}\right] \\
& +\left[\rho_{1}\|e\|\|r\|-k_{n} \rho_{1}^{2}\|r\|^{2}\right] .
\end{aligned}
$$

Completing the squares of the terms in the brackets, we obtain

$$
\dot{V} \leq-\left[1-\frac{1}{2 k_{n}}\right]\|e\|^{2}-\left[1-\frac{1}{4 k_{n}}-\frac{1}{4 k_{n}}\|r\|^{2}\right]\|r\|^{2}-\|s\|^{2},
$$

which using the definition of $x(t)$ of Eq. (45) can be further upper bounded $\dot{V}(t)$

$$
\dot{V} \leq-\left[1-\frac{1}{2 k_{n}}\left(1+\|x\|^{2}\right)\right]\|x\|^{2}
$$


The sign of the upper bound of $\dot{V}(t)$ is determined by the term in the brackets of Eq. (49). This term has to be positive to ensure the negative semi-definiteness of $\dot{V}(t)$, that is to ensure the negative semi definiteness of $\dot{V}(t)$, we must have

$$
1-\frac{1}{2 k_{n}}\left(1+\|x\|^{2}\right)>0
$$

for $\dot{V}(t)$ to be negative semi-definite. From Eq. (44), a sufficient condition on Eq. (50) can be obtained as

$$
1-\frac{1}{2 k_{n}}\left(1+\frac{V(t)}{\lambda_{1}}\right)>0
$$

and hence at this point, the analysis can be reformulated as

$$
\dot{V} \leq-\beta\|x\|^{2} \text { provided that } 2 k_{n}>\left(1+\frac{V(t)}{\lambda_{1}}\right),
$$

where $\beta$ is some positive constant $(0<\beta \leq 1)$. Due to the negative semi-defineteness of $\dot{V}(t)$, the maximum value that $V(t)$ can have is its initial value, $V(0)$, therefore, from Eq. (44), a more conservative condition on $k_{n}$ can be obtained to have the following form:

$$
\dot{V} \leq-\beta\|x\|^{2} \text { provided that } 2 k_{n}>1+\frac{\lambda_{2}}{\lambda_{1}}\|z(0)\|^{2},
$$

that is when $k_{n}$ is selected to satisfy Eq. (40), we can ensure that $V(t)$ Eq. (43) is bounded therefore $z(t) \in \mathcal{L}_{\infty}$ (i.e. $e(t), r(t), s(t)$ and $P_{0} \in \mathcal{L}_{\infty}$ ). Following standard signal chasing arguments, we can show that all signal in the closed-loop system are bounded and $e(t)$ and $\dot{\tilde{q}}(t)$ are uniformly continuous (from the boundedness of their derivatives over time), furthermore from the integration of both sides of Eq. (52), it is not difficult to see that $z(t), \in \mathcal{L}_{2}$ and therefore $e(t), \dot{\tilde{q}}(t) \in \mathcal{L}_{2}$. Finally, utilizing direct application of Barbalat's Lemma, ${ }^{19}$ we can obtain the result given in Eq. (38) provided that the gain condition of Eq. (40) is provided.

\section{Adaptive Controller Extension}

The controller formulation presented in the previous section assumed that the dynamical parameters of the robot manipulator are exactly known. In this section, in order to illustrate the flexibility of our core controller, we extend our result to an adaptive controller where the dynamical parameters of the robotic manipulator are assumed to be uncertain. We start our error system development for the adaptive controller by defining the parameter estimation error term as follows:

$$
\tilde{\theta} \triangleq \theta-\hat{\theta},
$$

where $\tilde{\theta}(t) \in \mathbb{R}^{p}$ represents the parameter estimation error vector, and $\hat{\theta}(t) \in \mathbb{R}^{p}$ represents a dynamic estimate of $\theta$.

Following a similar approach to our core controller the control input torque signal, $\tau(t)$, for the adaptive version is formed as

$$
\tau=Y_{d} \hat{\theta}+K_{p} e+K_{c} \alpha\left(q_{d}-\hat{q}\right)+K_{c}\left(\dot{q}_{d}-\dot{\hat{q}}\right),
$$


where $K_{p} \in \mathbb{R}^{n \times n}$ is a diagonal positive define control gain matrix and the parameter estimate vector $\hat{\theta}(t)$ is generated according to the following update rule:

$$
\begin{aligned}
\hat{\theta}= & \operatorname{Proj}\left\{\Gamma \left(Y_{d}^{T} e-\int_{0}^{t} \frac{d}{d \sigma}\left\{Y_{d}^{T}(\sigma)\right\} e(\sigma) d \sigma\right.\right. \\
& \left.\left.+\alpha \int_{0}^{t} Y_{d}^{T}(\sigma) e(\sigma) d \sigma\right)\right\}
\end{aligned}
$$

with $\Gamma \in \mathbb{R}^{p \times p}$ being a constant, diagonal, positive-definite, adaptation gain matrix, and $\operatorname{Proj}\{\cdot\}$ is a projection operator introduced to ensure the boundedness of $\hat{\theta}(t)$ and its time derivative. Note that similar to the re-arrangement presented in Eqs.(18), the Eq. (54) can be written in the following advantageous form:

$$
\tau=Y_{d} \hat{\theta}+K_{p} e+K_{c} r+K_{c} s .
$$

Similarly, after taking the time derivative of Eq. (55), the parameter update law can be re-written in the following form:

$$
\dot{\hat{\theta}}=\operatorname{Proj}\left\{\Gamma Y_{d}^{T} r\right\} .
$$

The observer formulation given in Eq. (13) is valid the adaptive controller formulation with a slight change in the the auxiliary bounding terms. That is; Eq. (21) becomes

$$
N_{0}=N_{d a}+N_{b a},
$$

where the auxiliary variables for the adaptive version of the controller $N_{d a}(t) \in \mathbb{R}^{n}$ and $N_{b a}(t) \in \mathbb{R}^{n}$ are defined as

$$
N_{d a}(t) \triangleq \ddot{q}_{d}+M^{-1}\left(q_{d}\right) Y_{d} \tilde{\theta}
$$

and

$$
\begin{aligned}
N_{b a}(t) \triangleq & \left(M^{-1}(q)-M^{-1}\left(q_{d}\right)\right) M\left(q_{d}\right) \ddot{q}_{d} \\
& +\left(M^{-1}(q)-M^{-1}\left(q_{d}\right)\right) Y_{d} \tilde{\theta} \\
& +M^{-1}(q)\left\{V_{m}\left(q_{d}, \dot{q}_{d}\right) \dot{q}_{d}-V_{m}(q, \dot{q}) \dot{q}\right. \\
& +G\left(q_{d}\right)-G(q)+F_{d}\left(\dot{q}_{d}-\dot{q}\right) \\
& \left.+K_{p} e+K_{c}(r+s)\right\} .
\end{aligned}
$$

We would like to highlight that the projection algorithm used in Eq. (55) is used to ensure that the bounding arguments given in Remark 1 are also valid for $N_{d a}$ and $N_{b a}$. Substituting the adaptive controller Eq. (54) into Eq. (33), we obtain the closed-loop dynamics for $r(t)$ as follows:

$$
M(q) \dot{r}=-V_{m}(q, \dot{q}) r+\chi-K_{c} r-K_{c} s-K_{p} e+Y_{d} \tilde{\theta},
$$

where $\tilde{\theta}$ term was defined in Eq. (53).

Having obtained the closed-loop dynamics for the observation error and the tracking error systems, we are now ready to propose the following theorem.

Theorem 2. The adaptive version of the controller proposed in Theorem 1, that is the controller of Eq. (54) with the update law Eq. (55) ensure that the closed-loop observer/controller is semi-globally asymptotically stable in the sense that

$$
\lim _{t \rightarrow \infty} e(t), \dot{\tilde{q}}(t)=0,
$$

provided that the controller and observer gains are selected to satisfy the gain conditions given in Eqs.(39) and (40). 
Proof. We start our proof by introducing the following non-negative function in the form

$$
V_{a}=V+\frac{1}{2} \tilde{\theta}^{T} \Gamma^{-1} \tilde{\theta}
$$

where $V(t)$ was defined previously in Eq. (43). Similar to the proof given in Theorem 1, $V_{a}(t)$ of Eq. (63) can be upper and lower bounded in the following form:

$$
\lambda_{3}\|x\|^{2} \leq \lambda_{3}\|y\|^{2} \leq V_{a} \leq \lambda_{4}\|y\|^{2}
$$

where the auxiliary variable $y(t)$ is defined as

$$
y(t) \triangleq\left[\begin{array}{lllll}
s^{T} & \sqrt{P_{0}} & r^{T} & e^{T} & \tilde{\theta}^{T}
\end{array}\right]^{T},
$$

the positive constants $\lambda_{3}, \lambda_{4}$ were defined in the following form:

$$
\begin{gathered}
\lambda_{3}=\min \left\{\lambda_{1}, \frac{1}{2} \lambda_{\min }\{\Gamma\}\right\} \\
\lambda_{4}=\max \left\{\lambda_{2}, \frac{1}{2} \lambda_{\max }\{\Gamma\}\right\} .
\end{gathered}
$$

with the positive constants $\lambda_{1}, \lambda_{2}$ were defined in Eq. (42). Differentiating Eq. (63) with respect to time, canceling common terms and following a similar procedure to that of Theorem 1 we can obtain

$$
\dot{V}_{a} \leq-\beta\|x\|^{2} \text { provided that } 2 k_{n}>\left(1+\frac{V_{a}(t)}{\lambda_{3}}\right),
$$

where the positive bounding constant $\beta$ was previously defined. From this point on following the exact analysis presented in Theorem 1, the boundedness of all closed-loop signals and asymptotic stability of the error signals can be proven provided the gain conditions are satisfied.

\section{Experimental Verifications}

In order to illustrate the feasibility and effectiveness of the proposed observer-based OFB adaptive tracking controller extensive experiments have been conducted on a two link revolute, direct-drive, planar robot manipulator build in our laboratory at Gebze Institute of Technology. The links of the manipulator are constructed from aluminium with the link lengths, $16 \mathrm{~cm}$ for the first link and $6.5 \mathrm{~cm}$ for the second link. Both joints are actuated via DC motors, a SanyoDemci motor equipped with a 4096 counts per revolution encoder for the first joint and an Escap motor equipped with a 1024 counts per revolution encoder for the second. Each joint motor is actuated by Copley Controls Corp Model 4122 DC motor amplifiers operated in torque control mode. The block diagram representation of the experimental setup is given in Fig. 1 and top and side views of the experimental setup is presented in Fig. 2.

Though some of the dynamical parameters of the manipulator used in our experiments could have been estimated, due to the existence of high frictional terms on the in-house build experimental set up, the adaptive version of the controller formulation is used in the experimental studies. The dynamics of the experimental setup is taken from a similar two link planer manipulator ${ }^{21}$ and is assumed to have the following form:

$$
\begin{aligned}
{\left[\begin{array}{l}
\tau_{1} \\
\tau_{2}
\end{array}\right]=} & {\left[\begin{array}{cc}
p_{1}+2 p_{3} c_{2} & p_{2}+p_{3} c_{2} \\
p_{2}+p_{3} c_{2} & p_{2}
\end{array}\right]\left[\begin{array}{l}
\ddot{q}_{1} \\
\ddot{q}_{2}
\end{array}\right]+\left[\begin{array}{cc}
-p_{3} s_{2} \dot{q}_{2} & -p_{3} s_{2}\left(\dot{q}_{1}+\dot{q}_{2}\right) \\
p_{3} s_{2} \dot{q}_{1} & 0
\end{array}\right]\left[\begin{array}{l}
\dot{q}_{1} \\
\dot{q}_{2}
\end{array}\right] } \\
& +\left[\begin{array}{cc}
f_{d 1} & 0 \\
0 & f_{d 2}
\end{array}\right]\left[\begin{array}{l}
\dot{q}_{1} \\
\dot{q}_{2}
\end{array}\right],
\end{aligned}
$$

where $p_{1}\left(\mathrm{~kg} \mathrm{~m}^{2}\right), p_{2}\left(\mathrm{~kg} \mathrm{~m}^{2}\right), p_{3}\left(\mathrm{~kg} \mathrm{~m}^{2}\right)$, are parameters related to the masses of the links and link lengths of the manipulator while $f_{d 1}$ and $f_{d 2}(\mathrm{Nms})$ are the viscous friction parameters, $c_{2}$ and $s_{2}$ are 


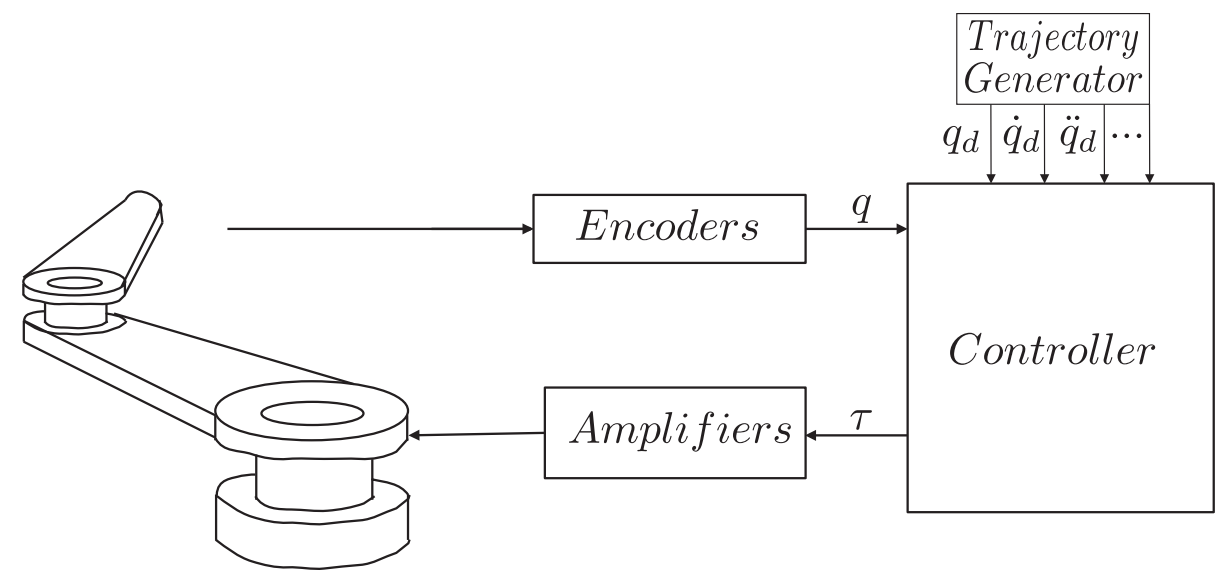

Fig. 1. The basic representation of the experimental setup.

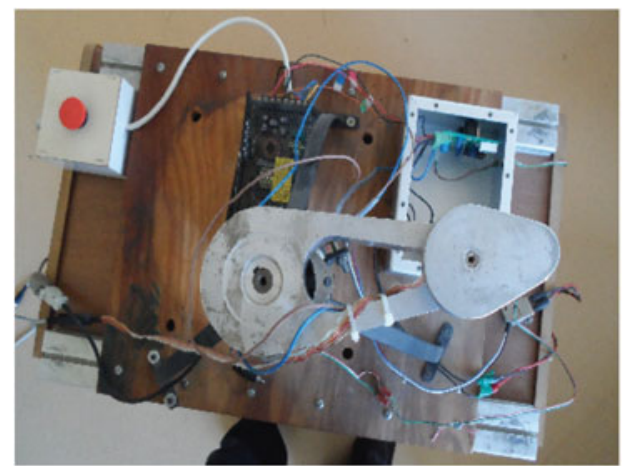

(a)

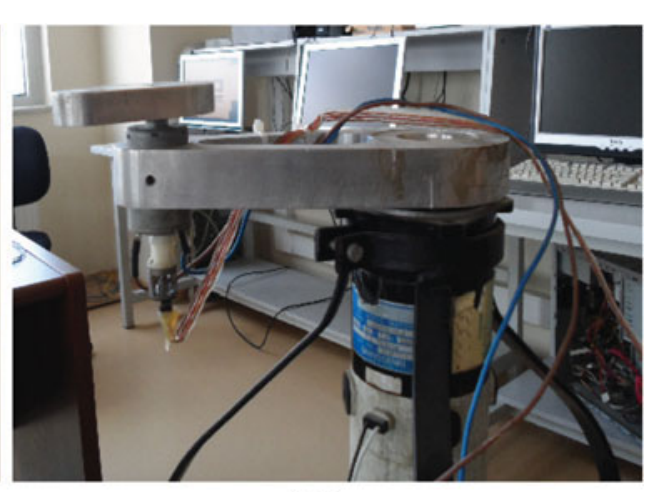

(b)

Fig. 2. The experimental setup.

used to denote $\cos \left(q_{2}\right)$ and $\sin \left(q_{2}\right)$ respectively. From Eqs.(7), (8), and the structure of Eq. (68), the uncertain parameter vector $\theta$ is obtained to have the following form:

$$
\theta=\left[\begin{array}{lllll}
p_{1} & p_{2} & p_{3} & f_{d 1} & f_{d 2}
\end{array}\right]^{T}
$$

During the experimental studies, the following desired link position trajectory were used

$$
q_{d}(t)=\left[\begin{array}{l}
0.7 \sin (2.5 t)\left(1-\exp \left(-0.3 t^{3}\right)\right) \\
1.2 \sin (2.5 t)\left(1-\exp \left(-0.3 t^{3}\right)\right)
\end{array}\right][\mathrm{rad}]
$$

where the exponential term was included to ensure that $\dot{q}_{d}(0)=\ddot{q}_{d}(0)=\dddot{q}_{d}(0)=0$. An illustration of the observer-controller controller with the parameter estimator used in the experimental studies is presented in Fig. 3. As illustrated, the controller implementation only requires the implementation of Eqs. (13), (54), and (55).

The initial link positions, velocities, and parameter estimate $\hat{\theta}(t)$ were initialized to zero. The observer-controller gains were tuned by trial-and-error until the a desirable link position tracking performance was achieved and are selected as

$$
\begin{aligned}
& \alpha=\operatorname{diag}\left\{\begin{array}{ll}
18 & 10
\end{array}\right\} \\
& K_{o}=\operatorname{diag}\left\{\begin{array}{ll}
25 & 300
\end{array}\right\}, K_{1}=\operatorname{diag}\left\{\begin{array}{ll}
300 & 300
\end{array}\right\}, \\
& K_{c}=\operatorname{diag}\left\{\begin{array}{ll}
75 & 80
\end{array}\right\}, K_{p}=\operatorname{diag}\left\{\begin{array}{ll}
1500 & 1800
\end{array}\right\}
\end{aligned}
$$




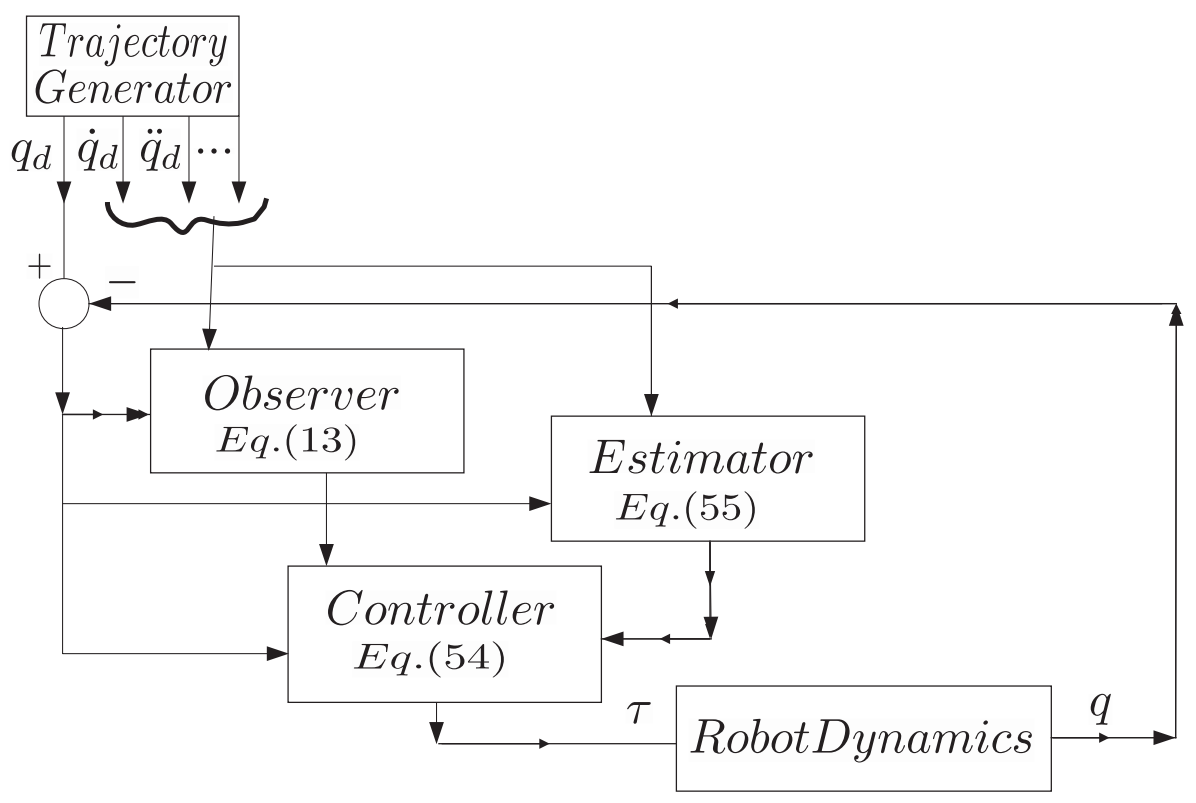

Fig. 3. Block diagram representation of experimental implementation.

with the adaptation gains set to

$$
\Gamma=\operatorname{diag}\{0.5,0.1,0.1,12,0.5\}
$$

The selection of controller and estimator gains were performed via trial and error. We start with the gains initially used in the simulation studies then change them by trial and error until a satisfactory tracking error performance is obtained.

Data acquisition and controller implementation were performed at a sampling frequency of $4 \mathrm{kHz}$ using a Quanser Q8 data acquisition board via a personal computer equipped with an Intel-based quad core central processor running Zenom, ${ }^{22}$ an in house real-time control environment that enables online gain tuning and graphical user interface. The link position tracking error is depicted in Fig. 4, while the corresponding voltage values of the control torque inputs are shown in Fig. 5. Also the parameter updates are presented in the Fig. 6. We also would like to note that the position tracking error values presented in Fig. 4 might seem high (around \pm 0.2 degrees). This is mainly due to the low resolution of the encoders used as link position sensors. To give an example, the encoder resolution of the second link used in the experimental studies is 1024 pulses for a revolution, which means for even with quadrature encoding each per pulse of the encoder only 0.09 degrees can be measured for the link. Due to this practical limitation, the errors in the experimental studies are high.

From Figs. 4 and 5, we can conclude that, around $20 \mathrm{~s}$. the tracking error performance within desirable limits with considerable acceptable control inputs and from Fig. 6, the parameter adaptations are completed around $50 \mathrm{~s}$.

\section{Conclusion}

In this work, a new model-free observer-based OFB tracking controller for robot manipulators have been presented. Specifically, a novel observer-controller couple was introduced that ensures semiglobally asymptotic the tracking despite the lack of link velocity measurements and uncertainty in the robot dynamics. Experimental studies are presented to illustrate the tracking performance of the observer-controller couple. The modular structure of proposed methodology seem to enable further extensions like robust and learning OFB controllers. 

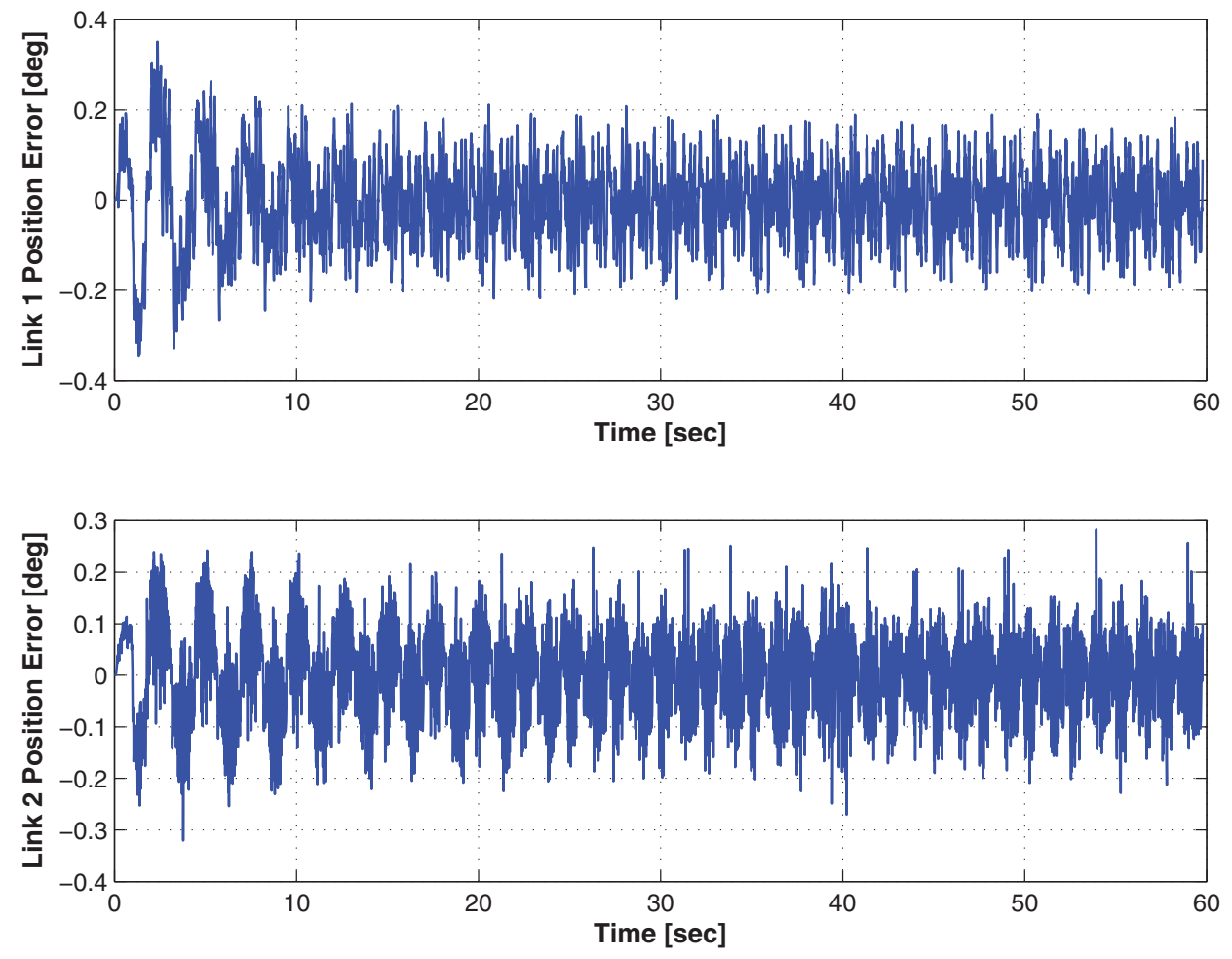

Fig. 4. Link tracking errors.
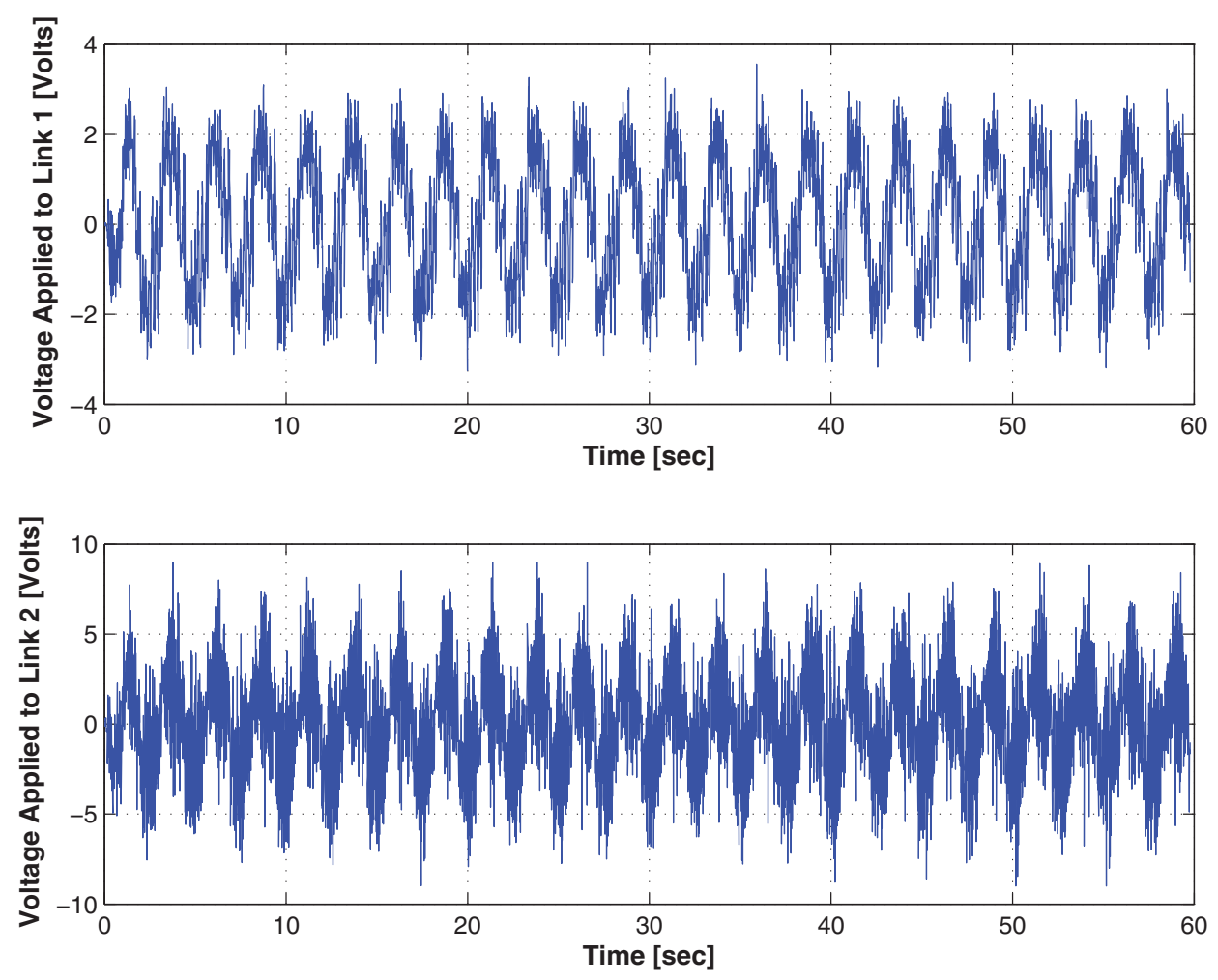

Fig. 5. Control torque inputs. 


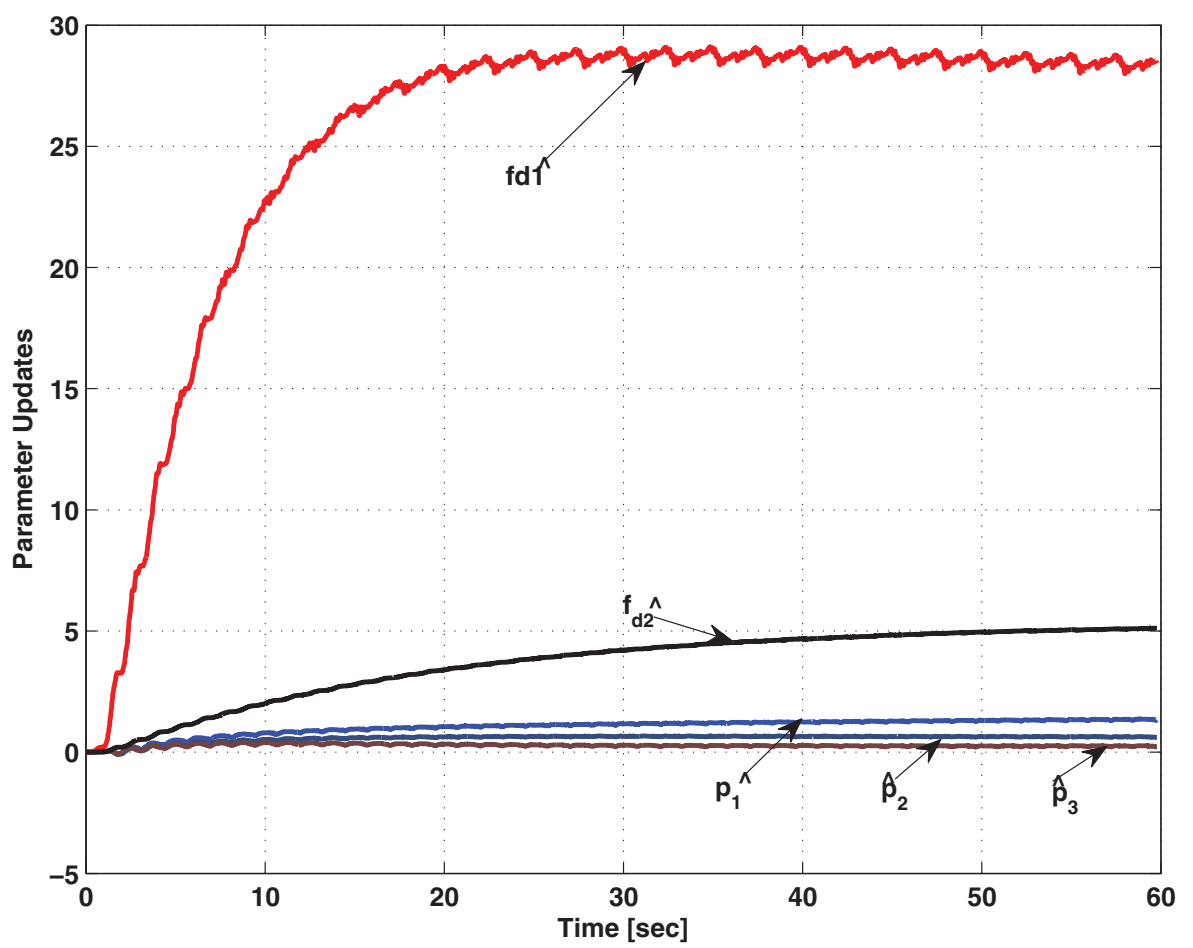

Fig. 6. Parameter updates.

\section{References}

1. S. Arimoto, V. Parra-Vega and T. Naniwa, "A Class of Linear Velocity Observers for Nonlinear Mechanical Systems," Proceedings of the Asian Control Conference, Tokyo, Japan (1994) pp. 633-636.

2. H. Berghuis and H. Nijmeijer, "A passivity approach to controller-observer design for robots," IEEE Trans. Robot. Autom. 9(6), 740-754 (1993).

3. T. C. Burg, D. M. Dawson and P. Vedagarbha, "A redesigned DCAL controller without velocity measurements: Theory and demonstration," Robotica 15, 337-346 (1997).

4. E. Zergeroglu, W. E. Dixon, D. Haste and D. M. Dawson, "A composite adaptive output feedback tracking controller for robotic manipulators," Robotica 17, 591-600 (1999).

5. J. Yuan and Y. Stepanenko, "Robust control of robotic manipulators without velocity measurements," Int. J. Robust Nonlinear Control 1, 203-213 (1991).

6. F. Zhang, D. M. Dawson, M. S. de Queiroz and W. E. Dixon, "Global adaptive output feedback control of robot manipulators," IEEE Trans. Autom. Control 45(6), 203-1208 (2000).

7. E. Zergeroglu, D. M. Dawson, M. S. de Queiroz and M. Krstic, "On global output feedback control of robot manipulators," IEEE International Conference on Decision and Control, Sydney, Australia (2000) pp. 5073-5078.

8. S. Nicosia and P. Tomei, "Robot control by using only position measurements," IEEE Trans. Autom. Control 35(9), 1058-1061 (1990).

9. C. Canudas de Wit and J. Slotine, "Sliding observers for robot manipulators," Automatica 27(5), 859-864 (1991).

10. S. Oh and H. K. Khalil, "Output feedback stabilization using variable structure control," Int. J. Control 62(4), 831-848 (1995).

11. A. Abdessameud and M. F. Khelfi, "A variable structure observer for the control of robot manipulators," Int. J. Appl. Math. Compt. Sci. 16(2), 189-196 (2006).

12. B. Xian, M. S. de Queiroz, D. M. Dawson and M. L. McIntyre, IOutput Feedback Variable Structure-like Control of Nonlinear Mechanical Systems," Proceedings of the 42nd IEEE Conference on Decision and Control, Maui, HA, USA (2003) pp. 368-373.

13. F. L. Lewis, C. T. Abdallah and D. M. Dawson, Control of Robot Manipulators (Macmillan Publishing Co., New York, USA, 1993).

14. P. Tomei, "Adaptive PD controller for robot manipulators," IEEE Trans. Robot. Autom. 7(4), 565-570 (1991).

15. E. Zergeroglu and E. Tatlicioglu, "Observer Based Output Feedback Control of Robotic Manipulators ," IEEE International Conference on Control Applications, Yokohama, Japan (2010) pp. 602-607.

16. E. Zergeroglu and E. Tatlicioglu, "Observer Based Adaptive Output Feedback Control of Robotic Manipulators ," IEEE International Conference on Decision and Control, Atlanta, GA, USA (2010) pp. $3638-3643$. 
17. N. Sadegh and R. Horowitz, "Stability and robustness analysis of a class of adaptive controllers for robot manipulators," Int. J. Robot. Res. 9(3), 74-92 (1990).

18. B. Xian, D. M. Dawson, M. S. de Queiroz and J. Chen, "A continuous asymptotic tracking control strategy for uncertain nonlinear systems," IEEE Trans. Autom. Control 47(7), 1206-1211 (2004).

19. M. Krstic, I. Kanellakopoulos and P. Kokotovic, Nonlinear and Adaptive Control Design (John Wiley \& Sons, New York, USA, 1995).

20. H. K. Khalil, Nonlinear Systems, 3rd ed. (Prentice Hall, New York, USA, 2002).

21. Direct Drive Manipulator Research and Development Package Operations Manual (Integrated Motion Inc., Berkeley, CA, USA, 1992).

22. C. Ay, H. Karakucuk, E. Kaleli and E. Zergeroglu, "Zenom: A Linux/Xenomai based Real Time Controller Implementation Platform," Proceedings of the 2014 Turkish Automatic Control Conference (TOK 2014), Kocaeli, Turkey (2014) pp. 201-206 (in Turkish). 